\title{
A Terahertz Quadruple-resonant Metamaterial using Single-particle Asymmetrical-ELC resonators
}

\author{
Minglei Shi ${ }^{\text {a }}$, Feng Lan, Ziqiang Yang, Yaxin Zhang, Zongjun Shi, Meng Li, \\ Hongen Su, Feng Luo and Qing Zhu
}

Terahertz Research Centre, School of Physical Electronics, University of Electronic Science and Technology of China

\author{
No.4, Section 2, North Jianshe Road, Chengdu 610054, P. R China \\ aemail: mlshi@std.uestc.edu.cn
}

Keywords: Metamaterials; Multi-band; Electric-LC resonators; Asymmetrical Coupling

\begin{abstract}
The design and simulation of an asymmetrical coupling quadruple-resonant bilayer terahertz metamaterial based on Electric-inductance capacitance (ELC) resonators are presented in this paper. The simulation results show that the resonant frequencies are $0.173,0.323,0.481$ and $0.622 \mathrm{THz}$, the first two of which are mainly caused by dipolar resonance and the other two are mainly caused by ELC resonance. Due to the asymmetrical electric field coupling mechanism, which is explained by the distributions of surface current, the resonant frequencies are designable by adjusting the corresponding geometrical parameters. Above all, the proposed structure can be applied in designing many multi-band terahertz devices.
\end{abstract}

\section{Introduction}

Artificial electromagnetic structures, known as metamaterials, can be engineered to obtain exotic electric and magnetic properties which can't be realized with natural materials [1]. Due to these novel properties, metamaterials research attracts intense attention of the scientists. Since Pendry has introduced the conception of split-ring resonators (SRRs) [2], various descendants have been derived, including electric-field-coupled resonator [3], chevrons shaped resonator [4] and other resonators [5] [6]. With the deepening of the research, the multi-band [7], high-Q (quality) [8], broadband [9], tunable [10] metamaterials can be realized by designing different structures, which are valuable in many electromagnetic engineering applications [11] [12]. Particularly, the multi-band metamaterials are widely used, such as absorbers [13], modulators [14] and polarizers [15], hence, it's attractive to design and fabricate multi-resonant metamaterials.

Recently, multi-band metamaterials have been designed and fabricated at microwave frequencies, which have proved that they have potential value of application [16]. Afterwards, with the development of terahertz, multi-band metamaterials in terahertz are intriguing with great flexibilities of spectrum resources [17]. Particularly, in terahertz region, Yuan et al. [18] have designed and fabricated a dual-band metamaterial using two different sized SRRs. The dual-band ELC-resonator which has two transmission minima at 0.92 and $1.39 \mathrm{THz}$ is composed of two single-resonance ELC that have the same capacitor-like gaps but different inductive loops. Due to the coupling between the different single-resonance ELCs within the structure, the transmission minimum and bandwidth of the first resonance are both enhanced.

In this paper, the design and simulation of an asymmetrical coupling quadruple-band metamaterial (ACQM) with four resonant frequencies at $0.173,0.323,0.481$ and $0.622 \mathrm{THz}$ are presented. The ACQM is composed of two different ELC resonators with different capacitor-like gaps and inductive loops which give birth to excite distinct dipolar and LC resonances. The two different single-particle resonators are designed in advance with a triple-resonance and dual-resonance, respectively. Compared with the individual resonators, the resonances of the integrated single-particle resonator, including the transmission minima and the relevant bandwidths, are obviously enhanced due to the mutual coupling between the different multi-resonant particles. 
Moreover, because of a resonant superposition, a total of five resonant peaks of two individual resonators decreased to four after integration.

\section{Design of ACQM}

A triple-resonant ELC resonator whose resonant frequencies are $0.213,0.478$ and $0.632 \mathrm{THz}$ and a dual-resonant ELC resonator whose resonant frequencies are 0.353 and $0.526 \mathrm{THz}$ are designed in advance, respectively. Then the two particles are integrated into a single particle ACQM. The bi-layer structure of the ACQM is made of aluminum with a thickness of $1 \mu \mathrm{m}$ and $170-\mu \mathrm{m}$-thick quartz substrate (relative permittivity is 4.41 and dielectric loss tangent is 0.0004 ). The dimension parameters of a unit cell are shown in Fig. 1(a). The stereogram of part of the ACQM is shown in Fig. 1(b).

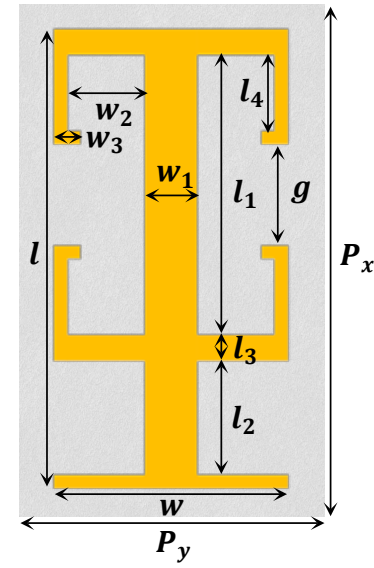

(a)

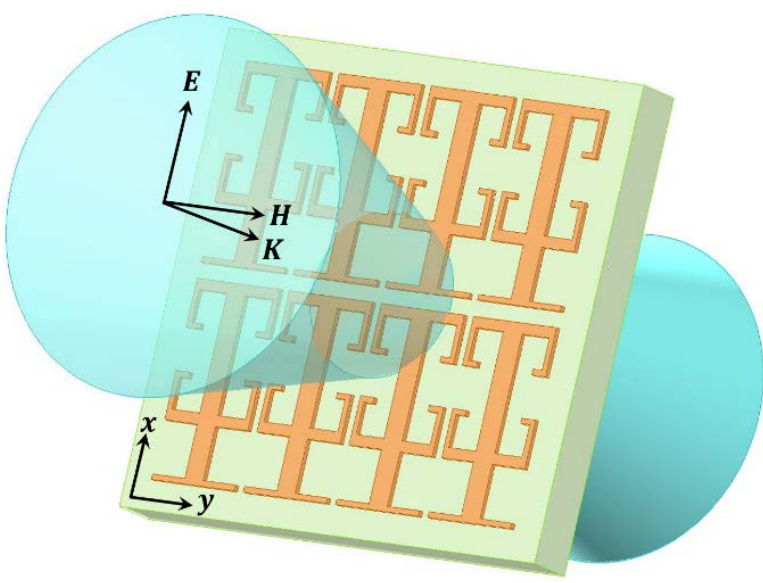

(b)

FIG. 1. (a) Geometrical parameters of unit cell: $l=360 \mu \mathrm{m}, w=180 \mu \mathrm{m}, l_{1}=220 \mu \mathrm{m}, l_{2}=100 \mu \mathrm{m}$, $l_{3}=20 \mu \mathrm{m}, l_{4}=60 \mu \mathrm{m}, g=80 \mu \mathrm{m}, w_{1}=40 \mu \mathrm{m}, w_{2}=60 \mu \mathrm{m}, w_{3}=20 \mu \mathrm{m}, P_{x}=400 \mu \mathrm{m}, P_{y}=200$ $\mu \mathrm{m}$ (b) Stereogram of part of the ACQM

\section{Results and Discussion}

The designs were all performed utilizing a finite element method (FEM) code. A unit cell simulation model was introduced to extract the transmission characteristics of the metamaterial. On the illuminated perpendicularly by TE mode incidence, the simulation result of the device is shown in Fig. 2, there are four transmission minima at $0.173,0.323,0.481$ and $0.622 \mathrm{THz}$ with low transmission of $-40.01,-34.31,-38.21$ and $-30.15 \mathrm{~dB}$, respectively, which presents a high performance filtering effect at terahertz band. Meanwhile there are three transmission peaks at $0.284,0.418$ and $0.545 \mathrm{THz}$ with transmission of $-1.24,-0.26$ and $-0.71 \mathrm{~dB}$. The bandwidths (BW) at $-10 \mathrm{~dB}$ of the four stopbands are $0.074,0.048,0.045$ and $0.029 \mathrm{THz}$, respectively. Generally, the $-10 \mathrm{~dB}$ bandwidths of the ACQM are significantly expanded compared with the single resonators.

In order to elucidate the physical mechanism of the asymmetrically mutual coupling between these multi-resonance, the upper triple-resonant particle, the lower dual-resonant particle and the total part of the quadruple-resonant particle, as illustrated in Fig. 3(a), are analyzed by full-wave simulations. And the simulation results are shown in Fig. 3(b).

By comparing the transmission of ACQM with the two individual particles ELC1 and ELC2, the four resonant frequencies for C1, C2, C3 and C4 show red shifts of 0.04, 0.03, 0.045 and $0.01 \mathrm{THz}$ compared with U1, L1, L2 and U3, respectively. On the other hand, the transmission minima, which of C1, C2 and C3, have a further decrease compared with U1, L1 and U2, L2, respectively. However, there exists a minor weakness at C4. The changes also reflected in the BW of stopbands. Remarkably, the first three resonance peaks C1, C2 and C3 have broader BWs compared with U1, $\mathrm{L} 1$ and U2, L2, the BW of C4 is $0.015 \mathrm{THz}$ narrower than which of U3 instead. Generally, the 
transmission minimum and bandwidth are both significantly enhanced compared with the proposed structure.

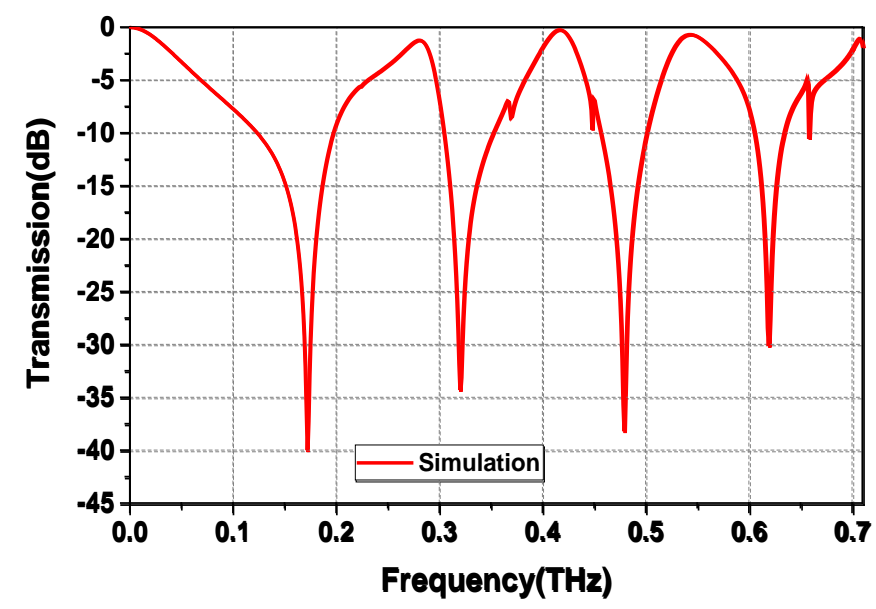

FIG. 2. Simulated transmission of ACQM in decibels.

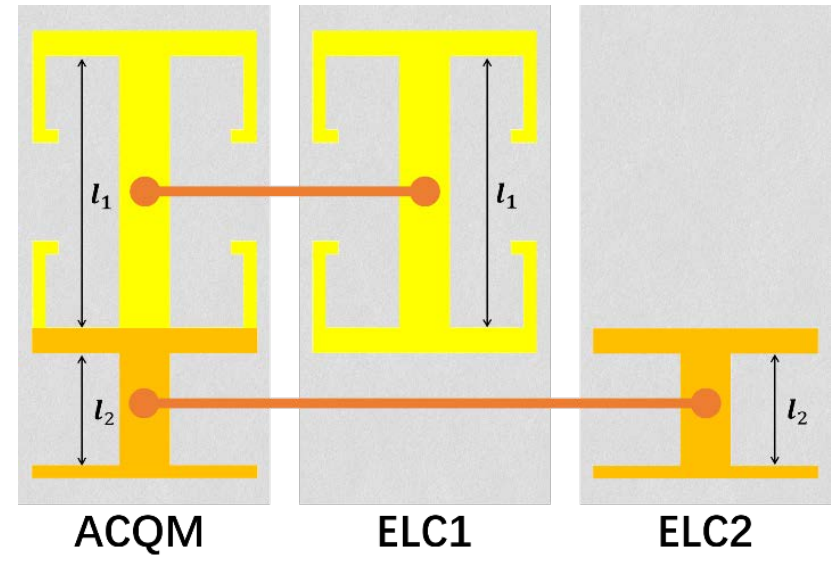

(a)

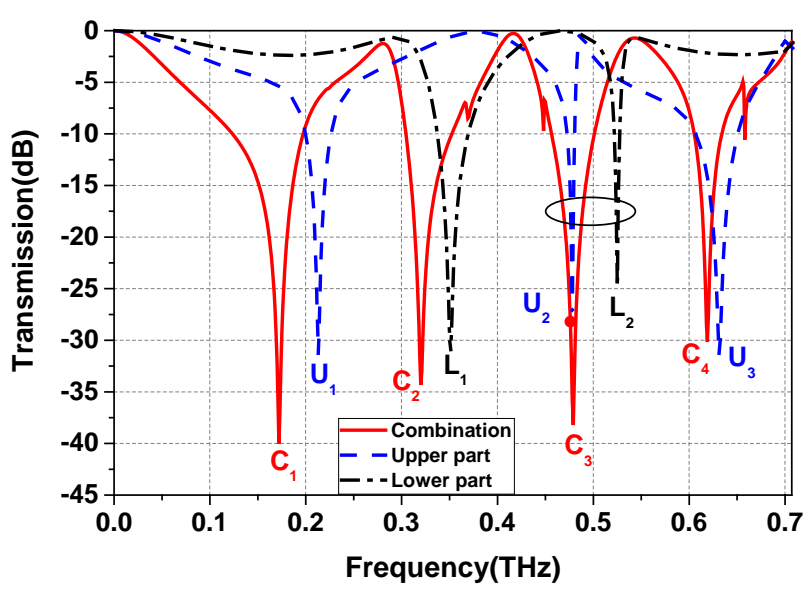

(b)

FIG. 3. (a) The combination part ACQM, the upper part ELC1 and the lower part ELC2 .(b) Transmissions of the ACQM (red solid line), ELC1 (blue dash line) and ELC2 (black dash dot line) in decibels. The four resonant peaks of ACQM are named C1, C2, C3 and C4. The three resonant peaks of ELC1 are named U1, U2 and U3. The two resonant peaks of ELC2 are named L1 and L2, respectively.

To better understand the asymmetrically mutual coupling effects on the resonance enhancement, the distributions of surface current are shown in Fig. 4 and Fig. 5, respectively.

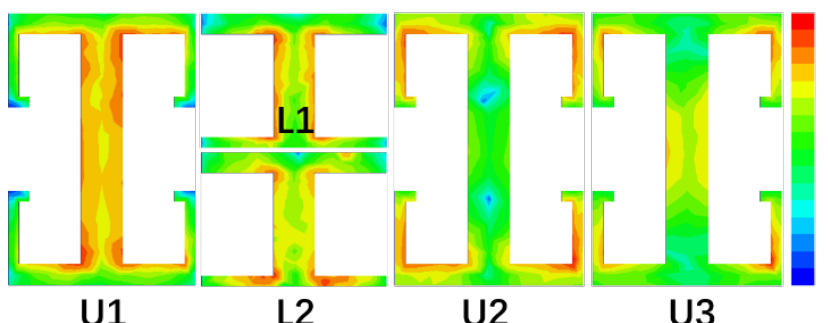

(a)

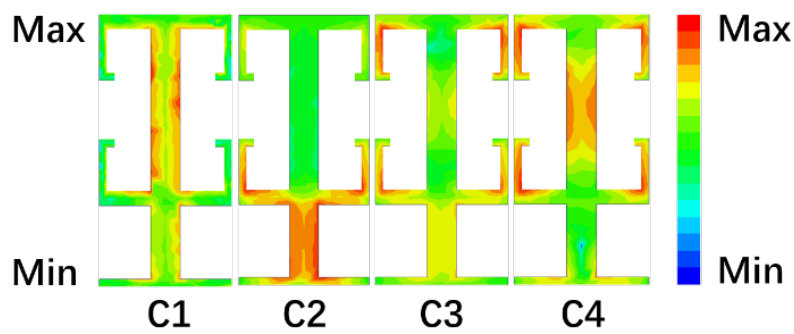

(b)

FIG. 4. (a). Amplitude of surface current distributions of ELC1 and ELC2 at their resonant frequencies. (b). Amplitude of surface current distributions of the ACQM at C1, C2, C3 and C4.

The amplitude of surface current distributions of ELC1 and ELC2 are shown in Fig. 4(a), respectively, suggesting that the different resonant peaks are corresponding to different structures. It can be found that U1 and L1, L2 are mainly caused by the electric dipolar response due to the 
stronger currents on the central arms of ELC1 and ELC2. Meanwhile, the currents are concentrated on the inductive loops indicating that $\mathrm{U} 2$ and $\mathrm{U} 3$ are mainly caused by the LC resonance. Furthermore, Fig. 4(b) shows the surface currents of ACQM for C1, C2, C3 and C4, respectively. One can see that there exist mutual coupling effects between the upper and the lower parts, which lead to the modest red shifts as shown in Fig. 3(b).

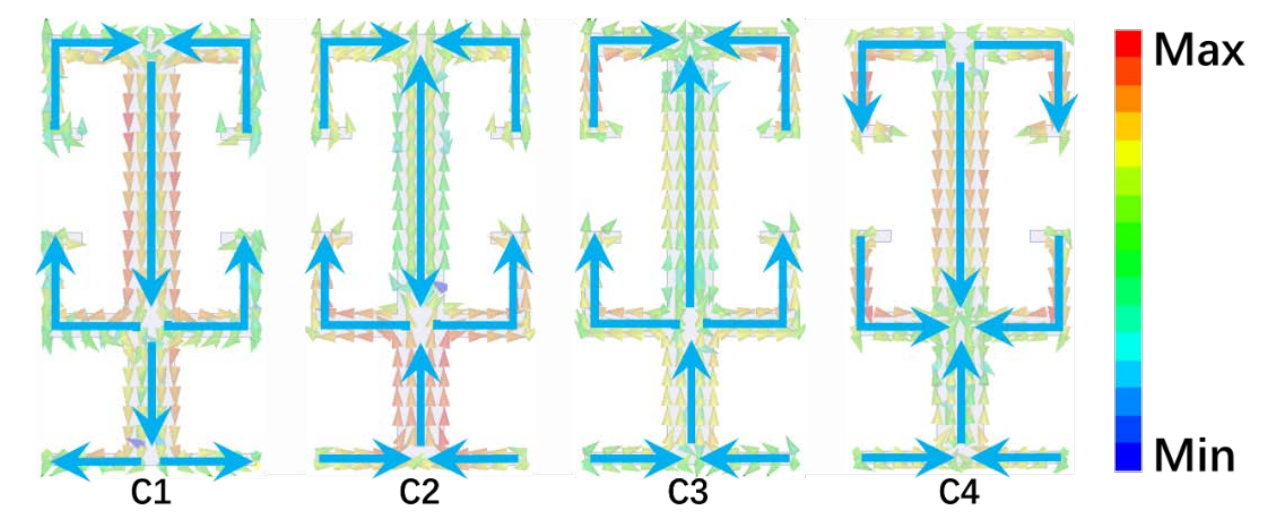

FIG. 5. Distributions of surface current vector of the ACQM at four different resonant frequencies.

To explain how the transmission and BW have been changed after integration, the distributions of current vector on surface of the particle for four resonant frequencies are demonstrated in Fig. 5, respectively. The currents on the upper portion and the lower portion of the central arm stream in the same direction for $\mathrm{C} 1$, which leads to two parallel dipole moments. As a result, the resonance is strengthened and the BW is expanded due to the coupling of the two dipole moments. The same physical mechanism occurred at C3, where a stronger resonance and a broader BW emerge because of the parallel dipole moments. For C2, coupling between the LC resonance of ELC1 with the electric dipole resonance of ELC2 strengthens the resonance also expands the BW. However, the currents on the upper portion and the lower portion of the central arm stream in opposite directions for $\mathrm{C} 4$, then the resonance is weakened and the BW become narrower because the particle electric dipole moments are partly counteracted.

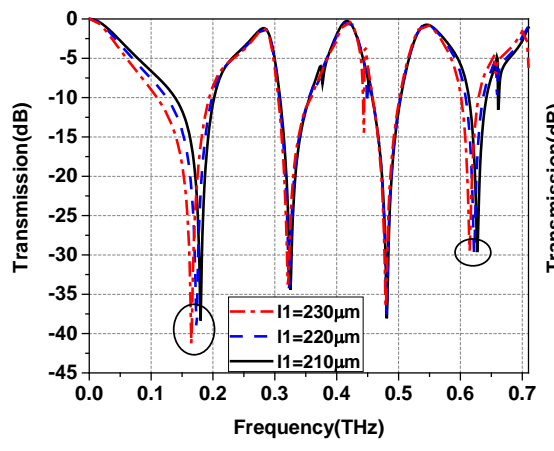

(a)

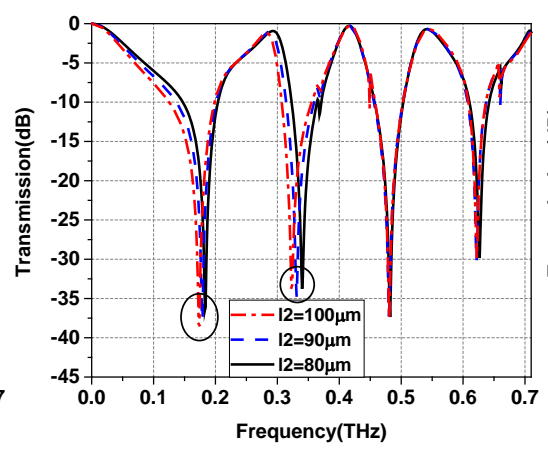

(b)

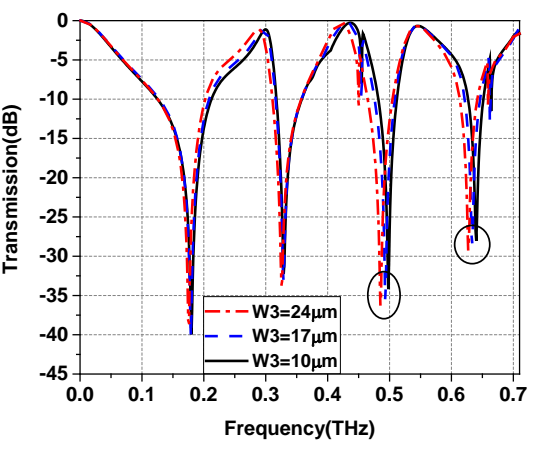

(c)

FIG. 6. Transmissions for variation of $l_{1}, l_{2}$ and $w_{3}$. (a) Variation of length $l_{1}$. (b) Variation of length $l_{2}$. (c) Variation of length $w_{3}$.

According to the above analysis, the resonant frequencies can be controlled by adjusting the relevant structure of the particle.

For the electric dipolar resonance, When $l_{1}$ which is the length of central arm of ELC1 is changed from 230 to $210 \mu \mathrm{m}$, the transmission of ACQM changes as Fig. 6(a) showing. It can be found that C1 and C4 have blue shifts while C2 and C3 are unaffected. For $l_{2}$ which is the length of central arm of ELC2, C1 and C2 show blue shifts while the C3 and C4 don't change when $l_{2}$ varies from 100 to $80 \mu \mathrm{m}$ as shown in Fig. 6(b). 
For the LC resonance, the resonant frequency $f=1 / \sqrt{L C}$. In this structure, $L$ and $C$ are corresponding to inductive loops and capacitor-like gaps, respectively. In this process of simulation, the gap width $w_{3}$ was changed from 24 to $10 \mu \mathrm{m}$ corresponding to the decrease of capacitance. From Fig. 6(c), it shows that there are blue shifts for C3 and C4, however, there almost no change for $\mathrm{C} 1$ and $\mathrm{C} 2$.

\section{Conclusion}

We present a design of a quadruple-band terahertz metamaterial based on asymmetrical ELC resonators. From the analysis of the current distributions on surface, it can be found that the first two resonant peaks are mainly caused by dipolar resonance and the other two peaks are mainly caused by LC resonance. Due to mutual coupling between the two parts of ACQM, three resonant peaks show stronger responses and broader BWs compared with the separated particles. The proposed structure can be applied in multi-band terahertz devices design.

\section{Acknowledgments}

This work was supported by the National Natural Science Foundation of China (No. 91438118), and the Fundamental Research Funds for the Central Universities of China (No. ZYGX2014J037 and ZYGX2014J033).

\section{References}

[1] D. R. Smith, J. B. Pendry, and M. C. K. Wiltshire, "Metamaterials and Negative Refractive Index," Science, vol. 305, pp. 788-792, 2004-08-06 00:00:00 2004.

[2] J. B. Pendry, A. J. Holden, D. J. Robbins, and W. J. Stewart"Magnetism from Conductors and Enhanced Nonlinear Phenomena." IEEE Transactions on Microwave Theory and Techniques, vol. 47, NO. 11, November 1999

[3] H. Odabasi, F. L. Teixeira, and D. O. Guney, "Electrically small, complementary electric-field-coupled resonator antennas," Journal of Applied Physics, vol. 113, p. 084903, 2013.

[4] N. Engheta, M. A. Noginov, N. I. Zheludev, N. Nandan, T. S. Saini, A. Kumar, et al., "Design and analysis of chevrons shaped split ring resonator in the mid-infrared region," vol. 9544, p. 95441D, 2015.

[5] Z. Yang, Q. Zhou, W. Long, C. Li, Y. Shi, A. Wu, et al., "Study of asymmetric U-shaped resonator at terahertz frequencies," Optics Communications, vol. 368, pp. 119-122, 2016.

[6] A. K. Horestani, Z. Shaterian, T. Kaufmann, and C. Fumeaux, "Single and dual band-notched ultra-wideband antenna based on dumbbell-shaped defects and complementary split ring resonators," in 2015 German Microwave Conference, 2015, pp. 64-67.

[7] L. Qi and C. Li, "Dual-band Frequency Selective Surface Bandpass Filters in Terahertz Band," Journal of the Optical Society of Korea, vol. 19, pp. 673-678, 2015.

[8] Z. Li, H. Huang, and J. Bao, "X-band low phase noise push-push oscillator utilizing High-Qdifferential transmission line loaded with multiple split-ring resonator," Journal of Electromagnetic Waves and Applications, vol. 30, pp. 124-139, 2015.

[9] J. Tang, Z. Xiao, K. Xu, and D. Liu, "A polarization insensitive and broadband metamaterial absorber based on three-dimensional structure," Optics Communications, vol. 372, pp. 64-70, 2016.

[10]P. S. Taylor, E. A. Parker, and J. C. Batchelor, "An Active Annular Ring Frequency Selective Surface," IEEE Transactions on Antennas and Propagation, vol. 59, pp. 3265-3271, 2011.

[11]W. Withayachumnankul and D. Abbott, "Metamaterials in the Terahertz Regime," IEEE Photonics Journal, vol. 1, pp. 99-118, 2009. 
[12]Q. Le-Van, X. Le Roux, A. Aassime, and A. Degiron, "Electrically driven optical metamaterials," Nat Commun, vol. 7, p. 12017, 2016.

[13]Y. Cheng, R. Gong, and Z. Cheng, "A photoexcited broadband switchable metamaterial absorber with polarization-insensitive and wide-angle absorption for terahertz waves," Optics Communications, vol. 361, pp. 41-46, 2016.

[14]G. I. Kiani and T. S. Bird, "ASK modulator based on switchable FSS for THz applications," Radio Science, vol. 46, pp. n/a-n/a, 2011.

[15] Yongzhi Cheng ${ }^{1,2, *}$, Chenjun $\mathrm{Wu}^{2}$, Zhengze Cheng ${ }^{3}$, and Rongzhou Gong ${ }^{2}$," Ultra-Compact Multi-Band Chiral Metamaterial Circular Polarizer Based on Triple Twisted Split-Ring Resonator." Progress In Electromagnetics Research, vol. 155, 105-113, 2016

[16]O. Yurduseven, G. Turhan-Sayan, and A. E. Yilmaz, "Hybrid-shaped single-loop resonator: a four-band metamaterial structure," Electronics Letters, vol. 47, pp. 1381-1382, 2011.

[17]C. Y. Luo, Z. Z. Li, Z. H. Guo, J. Yue, Q. Luo, G. Yao, et al., "Tunable metamaterial dual-band terahertz absorber," Solid State Communications, vol. 222, pp. 32-36, 2015.

[18]Y. Yuan, C. Bingham, T. Tyler, S. Palit, T. H. Hand, W. J. Padilla, et al., "A dual-resonant terahertz metamaterial based on single-particle electric-field-coupled resonators," Applied Physics Letters, vol. 93, p. 191110, 2008. 